\title{
Optic nerve anatomy for future human biometric signature
}

\begin{abstract}
Purpose: Biometric system works on the basis of recognition of patterns. There are various existing biometric systems for identification. We present the anatomical morphology of optic nerve transverse section as one of the additional exclusive biometric signature in human beings.

Methods: Transverse optic nerve sections from enucleated eyes were studied after written consent. Enucleated specimens were received in laboratory for various indications and were documented.

Results: The septas of optic nerve, nerve bundle and inter-septal vascularisations in the optic nerve were unique for each transverse section.

Conclusion: In the study, optic nerve morphology was found to be an important biometric signature in humans.
\end{abstract}

Keywords: Anatomy, biometric, forensic, optic nerve, septas
Volume 8 Issue 2 - 2018

\section{Dipankar Das, Diva Kant Misra, Harsha Bhattacharjee, Ganesh Chandra Kuri, Panna Deka, Dhaval Patel, Prabhjot Kaur Multani, Anshul Singh, Apurba Deka \\ Department of ocular pathology, Sri Sankaradeva Nethralaya, India}

\begin{abstract}
Correspondence: Dipankar Das, Senior Consultant, Department of Ocular Pathology, Uveitis \& NeuroOphthalmology Services, Sri Sankaradeva Nethralaya, Beltola, Guwahati - 781028, Assam, India, Tel +0361-2228879, 23055I6, Fax \# 036I- 2228878,Email dr_dasdipankar@yahoo.com
\end{abstract}

Received: December 27, 2017| Published: March 062018

\section{Introduction}

As a exceptional characteristic, we have different biometric indicators for each and every human being. ${ }^{1-5}$ Finger printing, poroscopy, iris and retinal vessels scans are already there and are established trait for unique identification. ${ }^{1}$ Some of the biometric indicators such as finger print or iris colour and configuration are modifiable. ${ }^{2,3}$ Optic nerve, as a cranial nerve is unique. It is the only cranial nerve that comes out of the cranial cavity, only nerve to be visualized directly by ophthalmoscope and only nerve that is part of the central nervous system. We have seen a characteristic pattern of anatomical arrangement of transected optic nerve along with the micro-vessels in the septas separating the fascicles of optic nerve, which could be a unique anatomical characteristic at the point of transverse slice. Out of 200 optic nerve sections studied, no one had similar features with one another.

\section{Objectives}

To present the findings of anatomical and morphological optic nerve transections as human biometric signatures. Methodology: This study was approved by institutional research and ethics committee.

\section{Study design}

Laboratory based, prospective and observational study

\section{Material and methods}

A total of $(n=200)$ transected optic nerve sections were studied. All the eyeball specimens were enucleated for various indications and after proper written consent. $10 \%$ neutral buffered formalin fixed optic nerve were measured by digital calipers (Mitutoyo absolute Digimatic, Japan), then they were processed in different graded of alcohol, xylene. Paraffin blocks were made thereafter. They were cut in 3 to 4 micron size in automated microtome (LEICA RM 2145(Germany)/MICRM HM 340E, Thermo-scientific, China). Baking of the tissue in slides was carried out followed by deparaffinization with xylene and graded alcohol. After hydration, the slides were dried in the Incubators. Different stains (Hematoxylin Eosin, Masson's Trichrome) were done; they were seen and documented under Axioskop 40 in AxioCam MRc camera, Germany. Optic nerves were observed for the following patterns: 1. Distribution of septas and 2. The blood vessels within the septas were shown (Figure 1) (Figure 2).
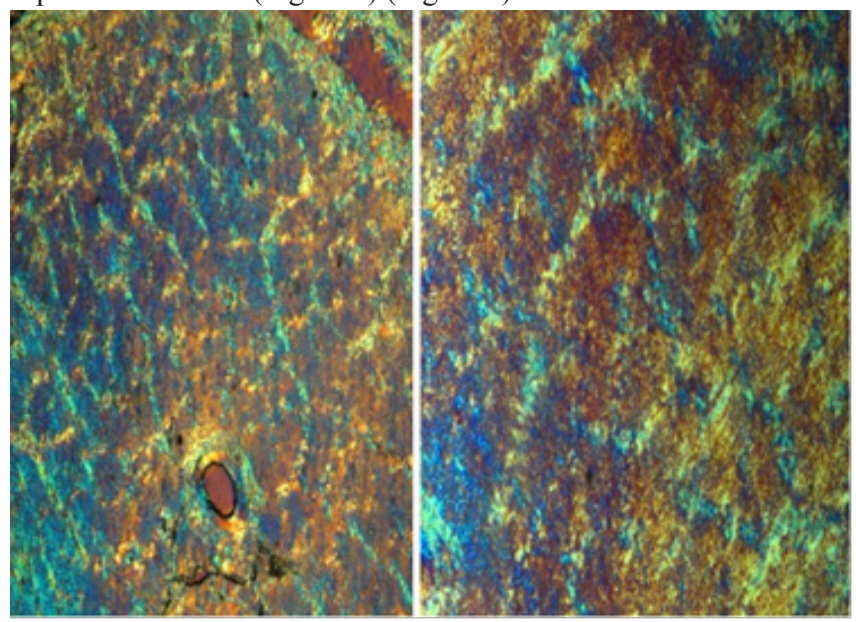

Figure I Transverse slice of optic nerve in diffraction and Hematoxylin and eosin (H\&E) Stain $(20 x, 40 x)$.

Some of the optic nerve sections were seen in unstained sections under the polarizer (Figure 3). Special stains such as Masson's trichrome were done to see the collagen septas containing the nerve bundles. There were various collagen types present in the optic nerves 
which was picked up by the trichrome stain. The mapping of optic nerve was documented in diffraction photography, using polarizer and different objective of compound microscope. Some of these images were rendered in gray scale and the white images on the dark background were adjusted to enhance the contrast. The images were analyzed uniformly based on magnifications (Figure 4). In this Figure, 15 optic nerve sections were shown without any similarity with one another in septas and vascular configurations. Out of 200 optic nerve sections, 57 optic nerve sections were documented in different magnifications. The optic nerves with tumour infiltration and inflammations were excluded from the cohort.

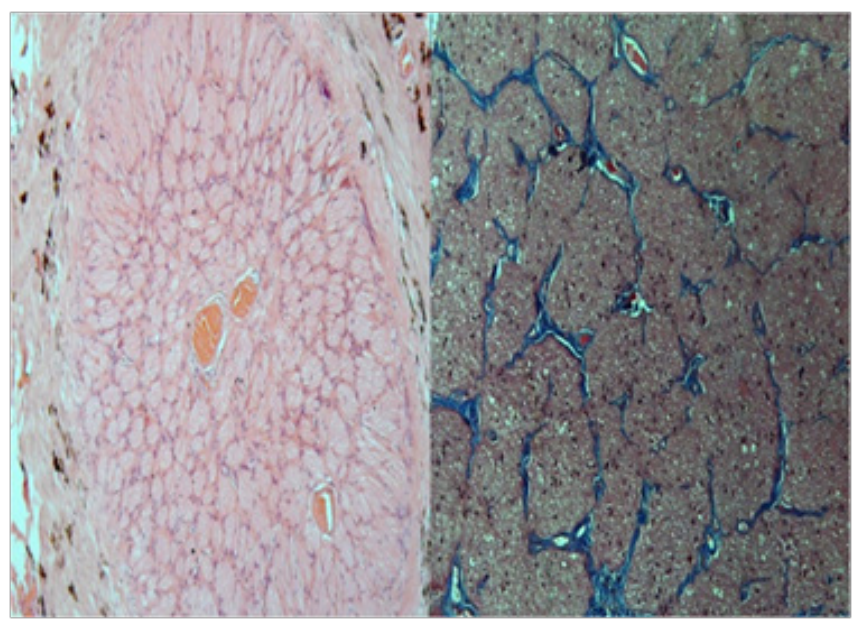

Figure $2 \mathrm{H} \& \mathrm{E}$ and Masson trichrome stain with septas and inter-septal blood vessels $(20 x, 40 x)$.

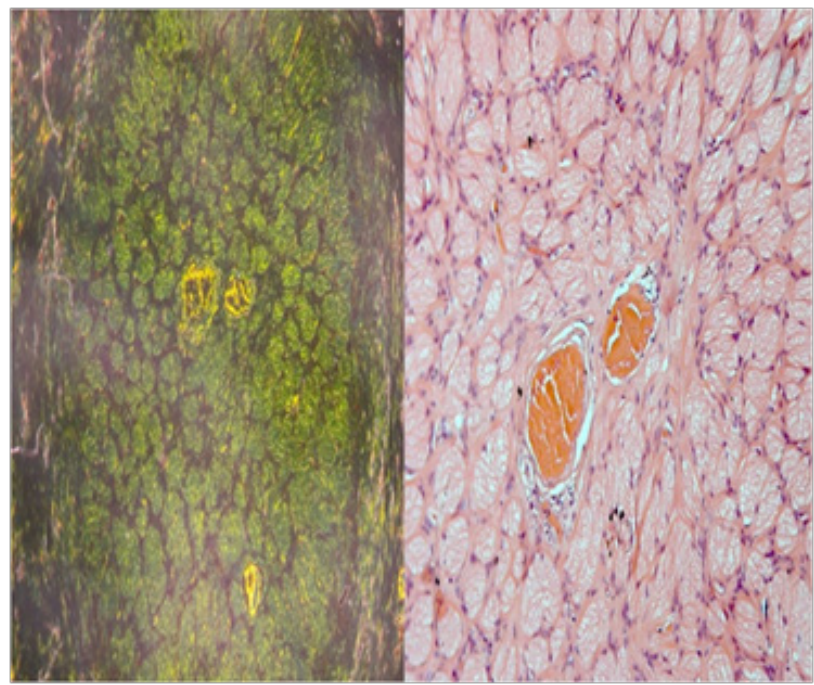

Figure 3 Optic nerve section of unstained slide, under polarizer (20x, 40x).

\section{Results}

The collagen septas of the optic nerve containing the bundles of nerves had unique distributions (Figures 1-4) and their orientations at the point of transverse cut of the optic nerves were exclusive. Optic nerve portion surrounded by glial tissue and the invading vessels were studied. In each septum, the arterial portion surrounded by the collagens which were clearly demarcated by trichrome stains was photographed. The dichotomous and anatomizing vascular plexus did not resemble same positional arrangement in other optic nerve sections. Size of the vessels in primary and secondary optic nerve septas also showed significant variations. No septal distributions in optic nerve had similar pattern with one another (Figure 4). The micro blood vessels within the collagen septas had a characteristic position distribution which did not match with any other optic nerve sections.

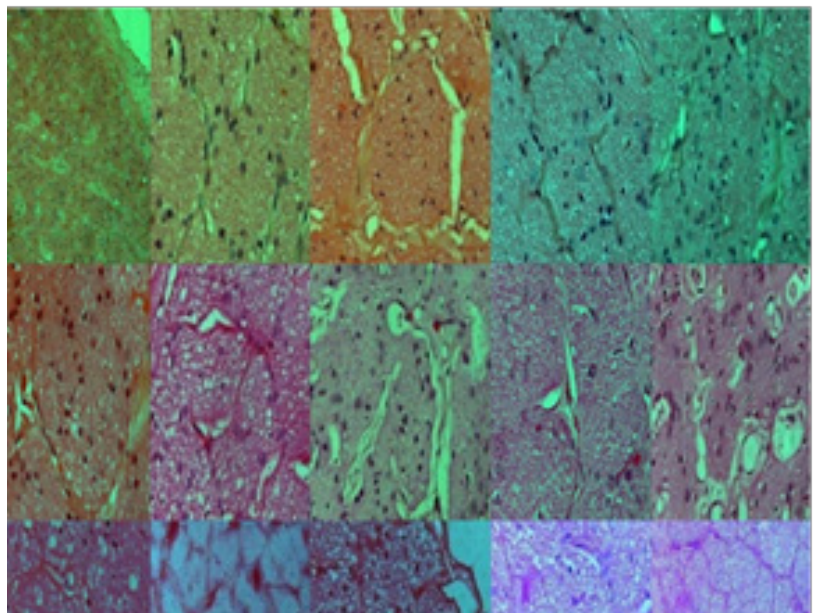

Figure 4 Different optic nerve sections showing fascicles with dissimilar septal distributions (H\&E, 20x, 40x)

\section{Discussion}

Optic nerve by itself is a distinctive cranial nerve and till now, in 'in-vivo imaging' to pick up the septas of the nerve and their nerve bundles seems to be remote. Anatomically, optic nerve is bounded by glia, that are passed into invading septal vessels in about fourth month of gestation. ${ }^{6-8}$ In its septum, a single artery enters surrounded by collagen tissue and neuroglia. These vessels divide dichotomously and anastomize with others forming a vascular plexus that reaches the middle of the nerves and the blood vessels. ${ }^{6,8,9}$ There may be six to nine thick main septas that divides the nerve into bundles and between many thinner minor septas, the blood vessels split frequently and dichotomously and rejoin it's others area to divide the optic nerve into fascicles. ${ }^{6-8}$ The inter-septal spaces are curved in human optic nerve and they are variable as singular distributions. The optic nerve configuration also varies in different mammals and other lower animals. ${ }^{6-10}$ In our study, most of the specimens were seen in the intraocular portion of the optic nerve as the glial septae between the fascicles present in the intraocular portion has much more characteristic features when compared to the orbital portion in some of the previous specimens. Anatomic characteristic uniqueness of the optic nerve at the point of slice is inimitable and it is unmatched with other optic nerve cut point visible under microscopy.

There are various biometric identifiers listed in human beings. Common identifiers are fingerprint recognition, face, voice, hand geometfry, signature variation, iris and retinal blood vessels recognition, thermograms, gait, keystroke, ear recognition, skin reflection, lip motion, body odor, patterns in tongue and most recently the DNA recognition. As we know from different biometric signatures of human being, the ophthalmic science has contributed two of its parts in this "identity-science", for instance, the iris pattern and retinal vessels configuration. Our thought process was for the third biometric mark in eye in the form of optic nerve pattern. The septas of optic nerve, at the point of specific cut is so variable that makes it a unique pattern. 
Second point which makes it more distinct is the position of blood vessels within the septas. If we compare it with the finger printing, then the finger print and the pores within them seems homologous to the optic nerve septas and blood vessels respectively. This makes the two structures distinctive. However, finger printing is external and can be modified or reduplicated but optic nerve is internal and cannot be reached to modify it. This can be unique for forensic study. In future, in-vivo vital staining with magnified stereoscopic imaging can be possible to demarcate those septas and the blood vessels within them. There are some imaging systems which can detect the septas or tracks in central nervous system imaging based on diffusion tensor magnetic resonance imaging for fiber tractography could be useful to explore in this situation. Since the cut sections of optic nerve which picked up the septas in histopathology are in around 3 micron size, modification of imaging based on echography such as echo planner imaging instead of spin echoes may also be helpful in "in-vivo imaging" of the optic nerve.

\section{Limitation of the study}

Optic nerve diseases like optic atrophy or papilloedema have an effect on this inimitable anatomical distribution of collagen septas and their blood vessels in the optic nerve. This might have an impact on reproducibility of these patterns during the process though they will have characteristic changes specific to the person and time. Additionally, effect of conditions like aging, nystagmus, progressive glaucoma, retinal vascular events and macular degeneration need to be evaluated. Due to lack of technology to study the internal features of optic nerve presently, it was difficult for us to study the effect of diseases and it's reversibility after the disease process has subsided.

\section{Conclusion}

Present study observed an inimitable anatomical distribution of collagen septas with their blood vessels in different fascicles, at the point of slice of the optic nerve. These anatomical optic nerve observations can be applicable when future in vivo imaging's methods can be developed to demarcate the collagen septas and intra-septal blood vessels.

\section{Acknowledgments}

Sri Kanchi Sankara Health and Educational Foundation, Guwahati, India.

\section{Conflicts of interest}

None.

\section{References}

1. Grorman L. Fingerprinting verification. In: Jain A, Bolle R, Pankanti S, editors. Biometrics Springer. New York, USA. 1999;44-48.

2. Hill R. Retinal identification. In: Jain A, Bolle R, Pankanti S, editors. Biometrics. Springer, New York, USA, pp.67-69.

3. Dunstone E. Lecture presented at; International Symposium on Signal Processing and its Applications (ISSPA), Kuala Lumpur. Malaysia. 2001

4. Maghiros I, Punie Y, Delaitre S, et al. Biometrics at the Frontiers: Assessing the Impact on Society. Seville: European Commission; 2005.

5. Trobe J, Leonello T. The Neurology of Vision. 1st ed. New York: Oxford University Press; 2001

6. Anderson D, Braverman S. Re-evaluation of the optic disc vasculature. Am J Ophthalmol 1976;82:165-168.

7. Anderson D, Hoyt W. Ultrastructure of intraorbital portion of human and monkey optic nerve. Arch Ophthalmol. 1969;82:506-508.

8. Anderson DR, Hoyt WF, Hogan MJ. The fine structure of the astroglia in the human optic nerve and optic nerve head. Trans Am Ophthalmol Soc. 1967;65:275-279.

9. Anderson D. Ultrastructure of human and monkey lamina cribrosa and optic nerve head. Arch Ophthalmol.1969;82:800-803.

10. Trainor PA, Tam PP. Cranial paraxial mesoderm and neural crest cells of the mouse embryo: co-distribution in the craniofacial mesenchyme but distinct segregation in the branchial arches. Development. $1995 ; 121: 2569-2582$. 